\title{
Autistic self-stimulatory behaviors (stims): Useless repetitive behaviors or nonverbal communication?
}

Article · June 2017

\section{CITATIONS}

0

2 authors:

\section{Leif Ekblad}

NA

6 PUBLICATIONS 6 CITATIONS

SEE PROFILE
READS

693

Some of the authors of this publication are also working on these related projects:

Project

Too precise or too imprecise - deciphering which parameter is gone awry in autism and psychosis View project

Project

Neurodiversity and relationships View project

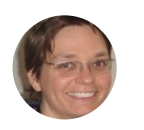

Gerit Pfuhl

University of Tromsø

48 PUBLICATIONS 201 CITATIONS

SEE PROFILE

Neurodiversity and relationships view project 
Autistic self-stimulatory behaviors (stims): Useless repetitive behaviors or nonverbal communication?

Leif Ekblad $^{1}$, Gerit Pfuhl²

1. Leif Ekblad, Independent researcher, Nöbbelöv 1005, 24193 Eslöv, Sweden.

2. Gerit Pfuhl, Department of Psychology, Uit The Arctic University of Norway

Keywords: Emotion, Repetitive behaviors, Stims, Autism, Neurodiversity, Communication 


\section{Abstract}

Background: Some of us engage in repetitive behaviors. Is this just a tick or do these repetitive behaviors, also knowns as stims serve a function? We here set out to test whether stims are part of non-verbal communication.

Methods: The study used the neurodiversity concept previously defined in Aspie Quiz (Ekblad, 2013) to research whether and how stims are related to emotions and feelings. A variety of stims were presented to 5,000 people and the relation to emotion words assessed.

Results: We extracted 22 stims that related to a wide range of emotions, such as being nervous, anxious, stressed, frustrated, anticipating, excited, angry, upset, closed state of mind and even pretending to be uninterested. Furthermore, the higher the score on neurodiversity (autistic tendencies) the more likely a person indicated using stims for expressing emotional states. Conclusion: Since the study shows that some stims are predictably related to emotions, we recommend that caregivers of nonverbal autistic children learn these associations which could facilitate to understand the child. 


\section{Introduction}

Repetitive behaviors are defined as part of the Autism Spectrum Disorders (ASD) and used as one of the two diagnostic criteria in DSM-V (American Psychiatric Association, 2013). Lam et al. (2008) found by using an explorative factor analysis that repetitive behaviors could be broken down into three categories: Repetitive Motor Behaviors, Insistence on Sameness, and Circumscribed Interests. The primary target of this study was Repetitive Motor Behaviors. A review of the literature by Leekam et al. (2011) concluded that restricted and repetitive behaviors had been relatively neglected in research studies. Gabriels et al. (2008) claimed, using correlations only, that repetitive and stereotyped behaviors were related to an unusual sensory profile. In the autistic community, Repetitive Motor Behaviors are typically called stims, a term that will be used in this study.

Rajagopalan \& Göcke (2014) proposed a visual method for identifying stims based on video sequences, with an aim to assist in autism diagnosis. Abowd et al. (2005) created a measuring device that could detect stims with an overall system accuracy of $69 \%$, although, the almost perfect detection accuracy of stimming events could be achieved by allowing for minor frame-level insertion errors. Bakan (2014) explored the link between stimming and music. Given that stims are a central part of autism, it is surprising that no hypothesis for the function of stims has been published, and that no empirical research for a possible function exists to date. Instead, the available research is focused on detecting and eliminating stims, and treatment plans typically try to extinguish stims without really knowing their potential function, something that could cause adverse long-term effects. 


\section{Neurodiversity and Aspie Quiz}

Neurodiversity sees "atypical neurological development as a normal human difference" (Jaarsma\&Welin, 2012), and hence the right to be different but not treated as ill or needing a cure (Jaarsma\&Welin, 2012, Griffin\&Pollak, 2009). Neurodiversity has been defined with factor analysis using the Aspie Quiz online questionnaire (Ekblad, 2013). The primary factor in Aspie Quiz was named neurodiversity factor while the secondary factor was named neurotypical factor. In the construction phase, 1,800 items were evaluated using a population of 550,000 individuals. Scores were calculated by using the factor loadings as weight factors. The difference between the neurodiverse score and the neurotypical score was used to provide classifications. A score difference above or equal to 35 was classified as neurodiverse (ND), a score difference below or equal to -35 was classified as neurotypical (NT), while scores in between were classified as mixed. The score difference had a .83 correlation with the AQ test measuring autistic traits (Ekblad, 2013).

\section{Aims and hypothesis}

In Aspie Quiz, factor analysis of stims and neurodiverse communication differences like staring, unusual emotion expression and tunes or rhythms constantly replaying themselves, cluster on the same factor. Unlike what some researchers have assumed (Gabriels et al. 2008), stims did not cluster with sensory traits (Ekblad 2013). This, though, makes it unlikely that stims can be explained by sensory differences. Hence, our hypothesis is that stims are a means of communication, i.e. the play a role in neurodiverse communication.

\section{Method}

Initially, a pilot study was set up in Aspie Quiz that presented 45 stims to neurodiverse participants (beta version 8, 2006). For each stim a drop-down menu offered 32 emotion words, or "I don't do this stim", or "non-emotional reason". Each participant could select up to three of 
the 32 possible emotions for each stim, or could select "I don't do this stim", or "non-emotional reason". Results from this pilot study indicated that to account for $50 \%$ of the answers for the preferred emotion, on average 4.6 emotions were required. For $80 \%$, on average 11.4 emotions were required and for $90 \%$, on average 16.0 emotions were required. That indicated that answers were not just random. Some of the emotions were associated with many stims (especially thinking, bored and anxious). This seemed to indicate that there were sometimes two reasons for doing a stim: a generic one and a specific one.

Driven by the success of the pilot study, we started an iterative process to find optimal stim - emotion associations. Here, we recruited a few hundred participants 1 . Stims that mostly appeared to relate to generic reasons, and for which no specific reason could be found, were dropped. To check the validity of the method, a few of the proposed associations were deliberately selected to be less likely.

Driven by the success of the pilot study, we started an iterative process to find optimal stim - emotion associations. Here, we recruited a few hundred participants 1 . Stims that mostly appeared to relate to generic reasons, and for which no specific reason could be found, were dropped. To check the validity of the method, a few of the proposed associations were deliberately selected to be less likely.

After the iterative pilot studies, a list of 22 promising stim - emotion pairs remained, see Table 1. These 22 stims - emotion pairs and one control with no known or suspected association (whistling when upset) were tested on a larger population (final version 2 items; H23, 2013). This stim survey was presented before the regular items in Aspie Quiz, and hence not to bias the answers by participants knowing their neurotype. Hence, this study is foremost about the function 
of stims, irrespective of whether one has few or many autistic traits and defines oneself as neurodiverse or neurotypical, respectively.

\section{Participants}

A total of 4,990 responses were collected (2,890 male and 2,100 female). Mean age was 31 years, SD 14 years. $54 \%$ of the participants were from the US, $13 \%$ were from the UK, and $6 \%$ were from Australia.

\section{Material}

The 22 stims from the iterative procedure and their emotion words were presented sequentially to the participants through the online survey system on rdos.net, see Table 1 for the stim - emotion pairs. The stims were presented with four response options: a) "I don't do this", b) a particular reason from the iterative pilot studies, c) "I do it independent of mood", and d) "I do it for other reasons". As an example: I flap my hands when I'm excited. The following analysis and results relate to this study.

\section{Data analysis}

First, the prevalence of "I don't do it" answers was compared to the prevalence of the three answers that stated that one does a particular stim. Second, the prevalence of "I do it independent of mood" was compared with the prevalence of the two emotional answer options "I do it for this reason" and "I do it for another reason". This is referred to as validity analysis. Third, to measure how representative the chosen emotion was, the prevalence of "I do it for another reason" was compared with "I do it for this reason" while excluding "I don't do it" and "I do it independently of mood" answers. This is an analysis of the emotional function. For all three analyses, we used a logistic regression model with the score difference from the Aspie Quiz as the predictor 


\section{Results}

The distribution of Aspie Quiz answers resulted in a grouping of 904 male as neurodiverse (ND), 1,161 male as neurotypical (NT), 949 female as neurodiverse and 602 female as neurotypical. The overall prevalence of stims was significantly higher in NDs (60\%, range $29 \%$ to $92 \%)$ than in NTs $(31 \%$, range $8 \%$ to $76 \%), \mathrm{t} 21=5.26, \mathrm{p}<.0001$, Cohen's $\mathrm{d}=1.6$. For those who do the stim, it expressed an emotion in both NDs and NTs and to a similar degree. There were 16 out of the 22 stims where NDs more often did it for the suggested reason, e.g. "jumping up and down when happy" than NTs did. The stims "pacing", "rubbing hands together", and "clenching fists" were statistically more often done for emotional reasons by NTs than NDs (though, note that these stims had a vast prevalence difference) but compared to prevalence the validity estimates had low to negligible effect sizes.

Table 1. Relation between emotions and stims.

\begin{tabular}{|c|c|c|c|c|c|}
\hline Stim & Reason/emotion & & Prevalence & $\begin{array}{c}\text { Validity/ Is } \\
\text { emotion }\end{array}$ & $\begin{array}{c}\text { Suggested } \\
\text { emotion }\end{array}$ \\
\hline \multirow{4}{*}{ Talking to yourself } & \multirow{4}{*}{ When hyperfocused } & ND & $92 \%$ & $54 \%$ & $69 \%$ \\
\hline & & NT & $76 \%$ & $63 \%$ & $57 \%$ \\
\hline & & $\mathrm{Z}$ & 13.2 & -4.9 & 5.1 \\
\hline & & $\mathrm{R} 2$ & .042 & .004 & .008 \\
\hline \multirow{4}{*}{ Walking on toes } & \multirow{4}{*}{ For pleasure } & ND & $48 \%$ & $67 \%$ & $62 \%$ \\
\hline & & NT & $22 \%$ & $70 \%$ & $38 \%$ \\
\hline & & $\mathrm{Z}$ & 18.5 & -1.6 & 7.9 \\
\hline & & $\mathrm{R} 2$ & .058 & .001 & .040 \\
\hline \multirow{4}{*}{ Tapping fingers } & \multirow{4}{*}{ When nervous } & ND & $85 \%$ & $55 \%$ & $75 \%$ \\
\hline & & NT & $64 \%$ & $59 \%$ & $57 \%$ \\
\hline & & $\mathrm{Z}$ & 16.3 & -2.9 & 8.0 \\
\hline & & $\mathrm{R} 2$ & .051 & .002 & .024 \\
\hline \multirow{4}{*}{ Biting lip, cheek or tongue } & \multirow{4}{*}{ When anxious } & ND & $82 \%$ & $62 \%$ & $84 \%$ \\
\hline & & NT & $56 \%$ & $67 \%$ & $70 \%$ \\
\hline & & $\mathrm{Z}$ & 18.3 & -3.1 & 6.7 \\
\hline & & $\mathrm{R} 2$ & .045 & .002 & .020 \\
\hline \multirow{4}{*}{ Grinding teeth } & \multirow{4}{*}{ When stressed } & ND & $60 \%$ & $72 \%$ & $79 \%$ \\
\hline & & NT & $34 \%$ & $81 \%$ & $69 \%$ \\
\hline & & $\mathrm{Z}$ & 17.0 & -5.1 & 4.6 \\
\hline & & $\mathrm{R} 2$ & .080 & .010 & .010 \\
\hline
\end{tabular}




\begin{tabular}{|c|c|c|c|c|c|}
\hline \multirow{4}{*}{$\begin{array}{l}\text { Watching a spinning, } \\
\text { blinking or glittering object }\end{array}$} & \multirow{4}{*}{ For pleasure } & ND & $71 \%$ & $74 \%$ & $82 \%$ \\
\hline & & NT & $27 \%$ & $80 \%$ & $76 \%$ \\
\hline & & Z & 26.6 & -2.9 & 3.3 \\
\hline & & $\mathrm{R} 2$ & .012 & .003 & .006 \\
\hline \multirow{4}{*}{ Pacing } & \multirow{4}{*}{ When thinking } & ND & $77 \%$ & $77 \%$ & $76 \%$ \\
\hline & & NT & $42 \%$ & $86 \%$ & $70 \%$ \\
\hline & & $\mathrm{Z}$ & 21.8 & -6.3 & 3.4 \\
\hline & & $\mathrm{R} 2$ & .080 & .014 & .004 \\
\hline \multirow{4}{*}{ Spinning an object } & \multirow{4}{*}{ For pleasure } & ND & $70 \%$ & $74 \%$ & $87 \%$ \\
\hline & & NT & $42 \%$ & $78 \%$ & $84 \%$ \\
\hline & & $\mathrm{Z}$ & 18.6 & -2.3 & 1.6 \\
\hline & & $\mathrm{R} 2$ & .055 & .002 & .001 \\
\hline \multirow{4}{*}{ Twirling hair } & \multirow{4}{*}{ For pleasure } & ND & $56 \%$ & $53 \%$ & $63 \%$ \\
\hline & & NT & $31 \%$ & $61 \%$ & $62 \%$ \\
\hline & & $\mathrm{Z}$ & 16.5 & -3.1 & 1.6 \\
\hline & & $\mathrm{R} 2$ & .042 & .003 & .002 \\
\hline \multirow{4}{*}{$\begin{array}{l}\text { Pulling hairs from head, } \\
\text { face or body }\end{array}$} & \multirow{4}{*}{ When frustrated } & ND & $53 \%$ & $57 \%$ & $56 \%$ \\
\hline & & NT & $28 \%$ & $57 \%$ & $30 \%$ \\
\hline & & $\mathrm{Z}$ & 16.4 & -0.2 & 8.2 \\
\hline & & $\mathrm{R} 2$ & .043 & 0 & .046 \\
\hline \multirow{4}{*}{ Rubbing arms or thighs } & \multirow{4}{*}{ When anxious } & ND & $67 \%$ & $73 \%$ & $81 \%$ \\
\hline & & NT & $25 \%$ & $75 \%$ & $59 \%$ \\
\hline & & Z & 25.6 & -1.7 & 7.5 \\
\hline & & $\mathrm{R} 2$ & .112 & .001 & .031 \\
\hline \multirow{4}{*}{ Rubbing hands together } & \multirow{4}{*}{ When anticipating } & ND & $62 \%$ & $64 \%$ & $65 \%$ \\
\hline & & NT & $27 \%$ & $77 \%$ & $59 \%$ \\
\hline & & $\mathrm{Z}$ & 22.5 & -6.8 & 2.5 \\
\hline & & $\mathrm{R} 2$ & .084 & .018 & .003 \\
\hline \multirow{4}{*}{ Flapping hands } & \multirow{4}{*}{ When excited } & ND & $36 \%$ & $76 \%$ & $77 \%$ \\
\hline & & NT & $8 \%$ & $84 \%$ & $69 \%$ \\
\hline & & $\mathrm{Z}$ & 22.6 & -2.7 & 2.9 \\
\hline & & $\mathrm{R} 2$ & .124 & .007 & .009 \\
\hline \multirow{4}{*}{ Sticking tongue out } & \multirow{4}{*}{ When hyperfocused } & ND & $35 \%$ & $81 \%$ & $71 \%$ \\
\hline & & NT & $15 \%$ & $88 \%$ & $54 \%$ \\
\hline & & $\mathrm{Z}$ & 16.0 & -4.1 & 4.5 \\
\hline & & $\mathrm{R} 2$ & .050 & .017 & .016 \\
\hline \multirow{4}{*}{ Pressing eyes } & \multirow{4}{*}{ When stressed } & ND & $61 \%$ & $81 \%$ & $83 \%$ \\
\hline & & NT & $24 \%$ & $86 \%$ & $74 \%$ \\
\hline & & $\mathrm{Z}$ & 23.0 & -3.2 & 4.6 \\
\hline & & $\mathrm{R} 2$ & .089 & .006 & .012 \\
\hline \multirow{4}{*}{$\begin{array}{l}\text { Clenching and unclenching } \\
\text { fists }\end{array}$} & \multirow{4}{*}{ When angry } & ND & $64 \%$ & $71 \%$ & $73 \%$ \\
\hline & & NT & $26 \%$ & $82 \%$ & $73 \%$ \\
\hline & & $\mathrm{Z}$ & 23.4 & -6.3 & 0.0 \\
\hline & & $\mathrm{R} 2$ & .091 & .016 & 0 \\
\hline \multirow[t]{2}{*}{ Rocking back-\&-forth } & For comfort & ND & $60 \%$ & $77 \%$ & $88 \%$ \\
\hline & & NT & $18 \%$ & $77 \%$ & $75 \%$ \\
\hline
\end{tabular}




\begin{tabular}{|c|c|c|c|c|c|}
\hline & & $\begin{array}{c}\mathrm{Z} \\
\mathrm{R} 2\end{array}$ & $\begin{array}{r}26.3 \\
.130\end{array}$ & $\begin{array}{c}0.1 \\
0\end{array}$ & $\begin{array}{l}\mathbf{5 . 6} \\
.024\end{array}$ \\
\hline \multirow{4}{*}{ Jumping up and down } & \multirow{4}{*}{ When happy } & ND & $38 \%$ & $83 \%$ & $83 \%$ \\
\hline & & NT & $12 \%$ & $93 \%$ & $69 \%$ \\
\hline & & Z & 19.1 & -4.1 & 4.2 \\
\hline & & $\mathrm{R} 2$ & .076 & .018 & .017 \\
\hline \multirow{4}{*}{ Biting yourself } & \multirow{4}{*}{ When anxious } & ND & $35 \%$ & $75 \%$ & $69 \%$ \\
\hline & & NT & $9 \%$ & $81 \%$ & $56 \%$ \\
\hline & & $\mathrm{Z}$ & 20.2 & -1.0 & 3.6 \\
\hline & & $\mathrm{R} 2$ & .093 & .001 & .012 \\
\hline \multirow{4}{*}{ Banging head } & \multirow{4}{*}{ When upset } & ND & $29 \%$ & $89 \%$ & $85 \%$ \\
\hline & & NT & $6 \%$ & $91 \%$ & $66 \%$ \\
\hline & & $\mathrm{Z}$ & 19.5 & -2.2 & 4.5 \\
\hline & & $\mathrm{R} 2$ & .101 & .010 & .027 \\
\hline \multirow{4}{*}{ Crossing arms } & \multirow{4}{*}{$\begin{array}{c}\text { When in closed state } \\
\text { of mind }\end{array}$} & ND & $82 \%$ & $60 \%$ & $75 \%$ \\
\hline & & NT & $61 \%$ & $63 \%$ & $65 \%$ \\
\hline & & Z & 15.3 & -1.8 & 4.7 \\
\hline & & $\mathrm{R} 2$ & .042 & .001 & .009 \\
\hline \multirow{4}{*}{ Pullling grass } & \multirow{4}{*}{$\begin{array}{l}\text { Pretending to be } \\
\text { uninterested }\end{array}$} & ND & $56 \%$ & $51 \%$ & $47 \%$ \\
\hline & & NT & $27 \%$ & $51 \%$ & $33 \%$ \\
\hline & & Z & 18.6 & 0.0 & 4.2 \\
\hline & & $\mathrm{R} 2$ & .056 & 0 & .013 \\
\hline
\end{tabular}

First column is the self-stimulatory behavior, second column is the presented emotion, fifth column indicates whether subjects did the behaviour for emotional reasons i.e. validity whereas last column shows inasmuch it is the suggested emotion. Proportion per group shown, $\mathrm{Z}$ value based on score difference, statistically significant $\mathrm{p}$ (Bonferroni-Holm corrected) in bold. $\mathrm{ND}=$ neurodiverse, $\mathrm{NT}=$ neurotypical, $\mathrm{R} 2=$ pseudo $\mathrm{R}^{2}$

\section{Discussion}

It was possible to extract 22 stims that related to a wide range of emotions, such as being nervous, anxious, stressed, frustrated, anticipating, excited, angry, upset, closed state of mind and even pretending to be uninterested. We found that stims had a higher prevalence in NDs than NTs also seen in medium to large effect sizes. Neurodiverse people agreed more that the presented emotion or internal state was related to the specific stim described. Our study cannot distinguish whether stims are unintentional emotional expressions or whether they are intentionally done for communicating one's emotional state. Goldman et al. (2009) found a high prevalence of stims in 
low-functioning ASD. This group has to express themselves more non-verbally than verbally compared with high-functioning autistics. Note, that many of those stims by Goldman et al. were related to stress and anxiety. In other words, low functioning autistics have these stims because they are stressed and anxious, not because these stims are diagnostic of neurodiversity or ASD. It would be interesting to compare it with deaf and blind people. They might as well rely on the similar stims to express anxiety and stress. We also found that sometimes two stims represent a similar emotional state, preventing any unambiguous mapping of a stim to emotion. It needs to be seen whether these are generational, cultural are purely individual differences.

\section{Conclusion}

Neurodiverse people express internal states non-verbally with specific stims more so than do neurotypical people. Awareness of stims as a mean to express emotional states can facilitate social interactions between NDs and NTs and reduce felt distressed in social interactions. We especially encourage investigating stims in a natural setting to advance how accurate and reliable the link is between stims as a mean for emotional communication in neurodiverse people.

\section{Compliance with Ethical Standards}

All procedures performed in studies involving human participants were in accordance with the the 1964 Helsinki declaration and its later amendments or comparable ethical standards. The Aspie Quiz survey was constructed in such a way that participants could not be identified.

Informed consent was obtained from all individual participants included in the study. Research items in Aspie Quiz could be skipped by selecting the “?” alternative (checked by default), and skipping them had no effect on scores. Participants were informed that some items might be for research only. 


\section{References}

1. Abowd G.D., Vadas K., Westeyn T., Starner T., Bian X. (2005) Recognizing Mimicked Autistic Self-Stimulatory Behaviors Using HMMs, Wearable Computers, 2005. Proceedings. Ninth IEEE International Symposium doi:10.1109/ISWC.2005.45

2. American Psychiatric Association. Diagnostic and statistical manual of mental disorders. 5th edn, text rev. Washington, DC: APA; 2013

3. Bakan M.B. (2014) The Musicality of Stimming: Promoting Neurodiversity in the Ethnomusicology of Autism MUSICultures . 2014 Vol. 41 Issue 2, p133-161. 29p.

4. Ekblad L. (2013) Autism, Personality, and Human Diversity: Defining Neurodiversity in an iterative process using Aspie Quiz. SAGE Open 3(3): 2158244013497722.

5. Gabriels R.L., Agnew J.A., Miller L.J, Gralla J., Pan Z., Goldson E., Ledbetter J.C., Dinkins J.P., Hooks E. (2008) Is there a relationship between restricted, repetitive, stereotyped behaviors and interests and abnormal sensory response in children with autism spectrum disorders? Research in Autism Spectrum Disorders Volume 2, Issue 4, October 2008, Pages 660-670 https://doi.org/10.1016/j.rasd.2008.02.002

6. Goldman S., Wang C., Salgado MW et al. (2009). Motor stereotypies in children with autism and other developmental disorders. Developmental Medicine \& Child Neurology 51: $30-38$.

7. Griffin E., Pollak D. (2009). Student experiences of neurodiversity in higher education: Insights from the BRAINHE project. Dyslexia 15(1), 23-41

8. Jaarsma P, Welin S (2012). Autism as a natural human variation: reflections on the claims of the neurodiversity movement. Health Care Analysis 20: 20-30 
9. Lam K.S., Bodfish J.W., Piven J. (2008) Evidence for three subtypes of repetitive behavior in autism that differ in familiarity and association with other symptoms. Journal Child Psychology Psychiatry 49(11): 1193-1200.

10. Leekam S. R., Prior M. R., Uljarevic, M. (2011) Restricted and repetitive behaviors in autism spectrum disorders: A review of research in the last decade. Psychological Bulletin Vol 137(4), Jul 2011, 562-593. http://dx.doi.org/10.1037/a0023341

11. Rajagopalan S.S, Göcke R. (2014) Detecting self-stimulatory behaviours for autism diagnosis IEEE International Conference on Image Processing 2014, DOI:

10.1109/ICIP.2014.7025294 